\title{
Transconjunctival Levator Aponeurosis Advancement without Resection of Müller's Muscle in Aponeurotic Ptosis Repair
}

\author{
Akihiro Ichinose ${ }^{*, 1}$ and Igal Leibovitch ${ }^{2}$ \\ ${ }^{I}$ Department of Plastic Surgery, Kobe University. 7-5-2 Kusunoki-cho, Chuo-ku, Kobe, Hyogo 650-0017, Japan \\ ${ }^{2}$ Division of Oculoplastic and Orbital Surgery, Department of Ophthalmology, Tel-Aviv Medical Center, Tel-Aviv \\ University, Tel-Aviv, Israel
}

\begin{abstract}
The transconjunctival levator aponeurosis advancement without resection of Müller's muscle enables repair of aponeurotic ptosis without the need for surgical exposure through a skin incision. This technique may be preferred by many patients who are reluctant to undergo ptosis surgery because of the possible skin scar and who do not present with excessive upper eyelid laxity. A successful outcome requires careful patients' selection, familiarity with the surgical anatomy of the everted eyelid, as well as with the possible complications. Special consideration should be given to factors such as determining the degree of aponeurosis advancement to accord with the height of the eyelid during surgery and to creating the desired shape and size of the "double eyelid" in Asians. From our experience, this surgical technique is effective in correcting levator aponeurotic ptosis and contributes to a quick recovery of the normal anatomy and function of the eyelid. This method should therefore be added to the arsenal of techniques for blepharoptosis repair.
\end{abstract}

Keywords: Aponeurotic ptosis, transconjunctival levator aponeurosis repair, Müller's muscle.

\section{INTRODUCTION}

We have recently described the transconjunctival levator aponeurosis repair without resection of Müller's muscle [1]. This technique, which is presented here, enables advancement of the levator aponeurosis, similar to the transcutaneous approach [2-5], but without the need for exposure through a skin incision. It is based on advancing the levator aponeurosis, while temporally detaching Müller's muscle from its insertion site, and is effective in correcting cases of aponeurotic ptosis with good or excellent levator muscle function. It maintains a normal anatomical contour of the upper eyelid, preserves its function, and allows for a quick recovery. By avoiding a skin incision, there is no external scarring and the operating time is shortened. This technique may therefore be preferred by many patients who are reluctant to undergo ptosis surgery because of the possible external scar. It should also be adopted by surgeons who prefer levator aponeurosis advancement [2-5] rather than transconjunctival resection of Müller's muscle for ptosis repair [6-9].

This review discusses the advantages and drawbacks of this technique, the criteria for patient selection, the description of the surgical procedure, and the possible complications.

\section{ADVANTAGES AND DRAWBACKS}

\section{Advantages}

1. The skin is not incised; therefore there is no risk of an external scar in the upper eyelid. This may encourage reluctant patients to undergo the operation.

*Address correspondence to this author at the Department of Plastic Surgery, Kobe University, 7-5-2 Kusunoki-machi, Chuo-ku, Kobe, Hyogo 650-0017, Japan; Tel: +81-78-382-6251; Fax: +81-78-382-6269;

E-mail: ichise@med.kobe-u.ac.jp
2. The operation is quicker, and so is the post-operative recovery time.

3. A natural-looking eyelid is attained. Neither the levator palpebrae superioris (LPS) muscle nor Müller's muscle are resected, thereby preserving their normal contraction ability which is conducive to the smoothness of eyelid opening and closure [5].

4. Avoiding resection of the muscles and the conjunctiva prevents postoperative entropion and eyelid lag.

\section{Drawbacks and Solutions}

1. One of the main issues in levator aponeurosis repair in ptosis surgery is the difficulty in determining the amount of aponeurosis that should be advanced and fixated to the tarsal plate, based on the height and contour of the eyelid during surgery $[5,6]$. This is possibly influenced by the reduced function of the levator muscle from the local anesthesia [6], or because of an increased tonus of the orbicularis oculi muscle [5]. This difficulty is also encountered in our method, and should therefore be taken into consideration during surgery. Although there are no methods to quantify the amount of the levator aponeurosis advancement in aponeurotic ptosis repair as there is for congenital ptosis repair [5], some surgeons use exteriorized sutures to adjust the height of the eyelid post-operatively $[2,6]$. For correcting mild ptosis, we advance the aponeurosis down to an extent depending on the evaluated function of the LPS muscle of the eyelid. The distance of the lower border of the LPS muscle from the upper border of the tarsus is $0-5 \mathrm{~mm}$, which depends on the function of the levator and the severity of the ptosis. This 
distance, however, may vary according to factors such as the response to phenylephrine, impedance to eyelid opening, globe size and race. The extent of the advancement of the aponeurosis is generally slightly greater than that in transcutaneous aponeurotic repair. In cases of over- or under-correction, the patient is usually followed up for at least 6 months postoperatively before any revision surgery is performed. The risk of over-correction lasting more than 6 months postoperatively is very small in the transconjunctival approach.

2. It is sometimes quite difficult to create the desired height and shape of the superior palpebral crease. In East Asia, the procedure that is widely used when the desired crease is not otherwise attainable is suture blepharoplasty without a skin incision $[10,11]$.

3. Manipulation of soft tissues, such as the orbicularis oculi muscle and several fat pads, is the key to fashioning a natural and beautiful eyelid with an aesthetically superior palpebral crease in patients with thick eyelids. This, we feel, is limited in transconjunctival aponeurotic repair, especially the dissection of the preseptal and the retro-orbicularis oculi fat (ROOF) [12]. The amount of tissue to be manipulated should be planned pre-operatively and resected carefully with precise knowledge of the anatomy.

\section{METHODS}

\section{Patient Selection}

Selection of patients who may benefit from this surgical technique is an essential step in a successful post-operative outcome. Suitable candidates are those patients with an aponeurotic ptosis with good or excellent LPS muscle function, no excessive upper eyelid laxity, a fine superior palpebral crease, and who are reluctant to have a skin incision. Patients with a significant degree of dermatochalasis of the eyelid are less suitable for this surgical method. Other factors such as brow ptosis or forehead laxity should also be taken into consideration.

Patients should be advised of the possibility of postoperative worsening of dermatochalasis and upper eyelid laxity as a result of brow descent, which is frequently seen after correction of ptosis. Ptosis surgery could be combined with browpexy or forehead lift, however, not in patients in whom a precise size or shape of the double eyelid is expected and in those who have a thick eyelid that requires reduction.

\section{Preoperative Examination}

All patients should undergo a thorough ophthalmic examination. The procedure is contraindicated in patients with dry eyes, corneal inflammation or corneal erosions. Systemic conditions such as diabetes and hypertension should be well controlled pre-operatively. The operation is preferably performed after discontinuation of anticoagulant medications; provided that the cessation is approved by the treating physician and that it does not increase risk of a systemic thrombotic event.

\section{The Surgical Procedures}

The outline of the transconjunctival levator surgery is presented in (Fig. 1). The operation is usually performed under local anesthesia. A local anesthetic solution $(1 \%$ lidocaine, $0.4 \%$ ropivacaine HCL, $0.8 \%$ sodium bicarbonate with 1:200000 epinephrine) is injected with a 30 gauge needle in the subcutis of the eyelid, which is then everted with traction sutures, and under the conjunctiva. The dissection is carried out with a microdissection needle or a pair of sharp-pointed monopolar forceps powered by an Ellman Surgitron ${ }^{\circledR}$ radio-frequency generator or by a highfrequency electrosurgical unit. The conjunctiva is incised 1 $\mathrm{mm}$ above the upper border of the tarsal plate (Fig. 2A). Müller's muscle is cut from the tarsus, leaving the $1 / 3$ lateral part intact. A very thin glossy fascia lies between Müller's muscle and the levator aponeurosis (Fig. 2B). The aponeurosis is dissected from the frontal aspect of the tarsus up to a level of 2-3 $\mathrm{mm}$ below the superior border of the tarsus and then incised horizontally (Fig. 2C) and dissected laterally in front of the preserved part of Müller's muscle. Dissection is further carried out to explore the orbital septum which is then incised, and the lower border of the levator muscle is verified. The suborbicularis fascia, which lies in front of the aponeurosis, is a helpful indicator of aponeurosis and is easily identified by its abundance of vessels and nerves. Excision of the orbicularis oculi muscle around the "double eyelid" crease line facilitates formation of a fine superior palpebral crease. When found excessive, some of the subseptal fat pad or the nasal fat pad is resected. Such manipulation of the tissues is completed before the aponeurosis is fixed to the tarsus. The aponeurosis is advanced down and fixed to the fronto-superior part of the tarsus with 3 or 4 stitches using 6-0 PDS II ${ }^{\circledR}$ (Fig. 2D). The height and the shape of the eyelid is checked with the patient in a sitting position, and the levator aponeurosis is fixed with the subcutaneous tissue around the superior palpebral crease with 3 or 4 stitches of 6-0 PDS II ${ }^{\circledR}$. Müller's muscle is reattached and sutured to the superior edge of the tarsal plate, without resecting or tucking it, using 7-0 Vicryl ${ }^{\mathbb{B}}$. All the sutures are buried into the eyelid to avoid any irritating contact with the surface of the eye. The 2 or 3 sutures, which are passed from the superior tarsal edge and conjunctiva through the eyelid to the skin on the superior crease (Fig. 2E), are removed 5 days - 1 month postoperatively. Minor adjustments are made in the height of the superior eyelid crease by timing the removal of the sutures. A representative case is exhibited in (Fig. 3).

\section{DISCUSSION}

The transconjunctival approach for ptosis repair, which is presented here, is favored for being minimally invasive, not requiring a skin incision and for allowing a quick postoperative recovery.

With the rising number of patients with aponeurotic blepharoptosise, this eyelid pathology becomes widely recognized as a major cause of reduction in the quality of life. Although the surgical repair is not complex, many patients choose not to have surgery because of the eyelid skin incision. Although the eyelid scar in the transcutaneous approach tends to become inconspicuous, it may still be 


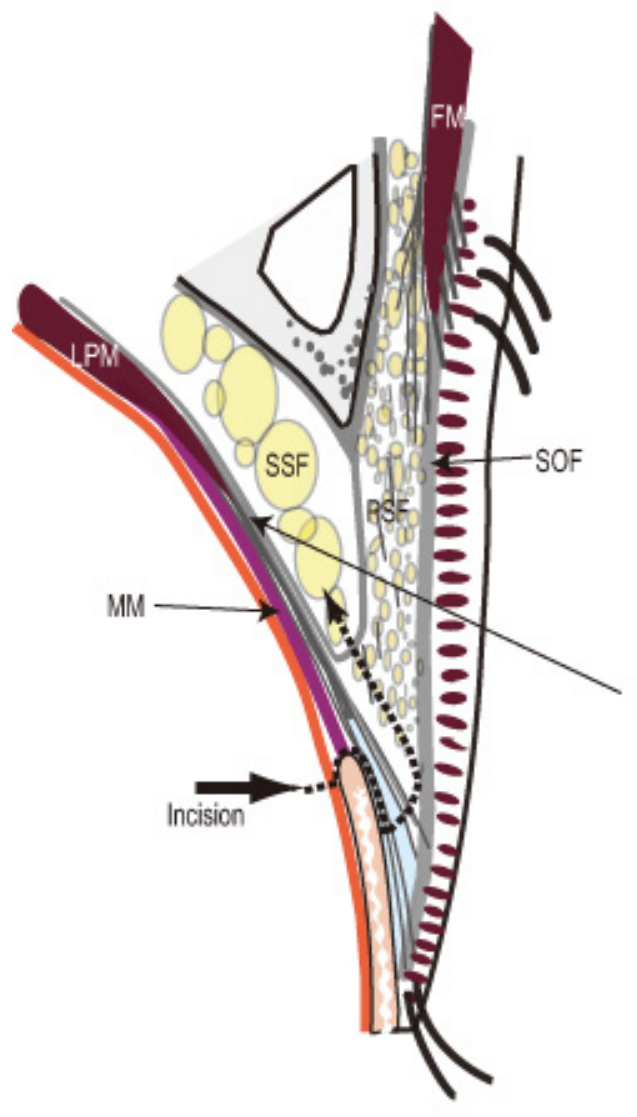

(A)

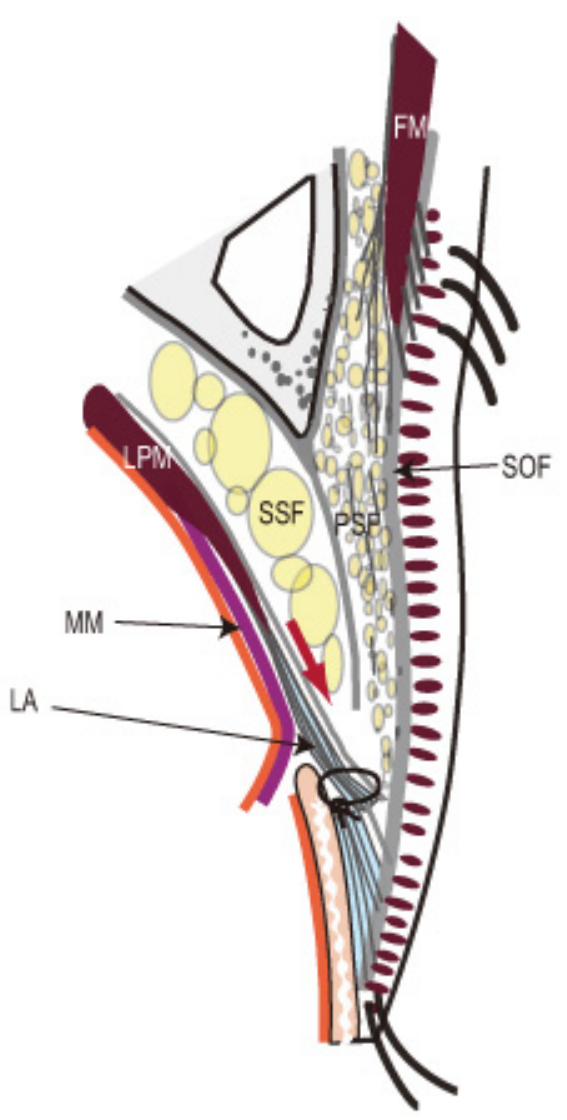

(B)

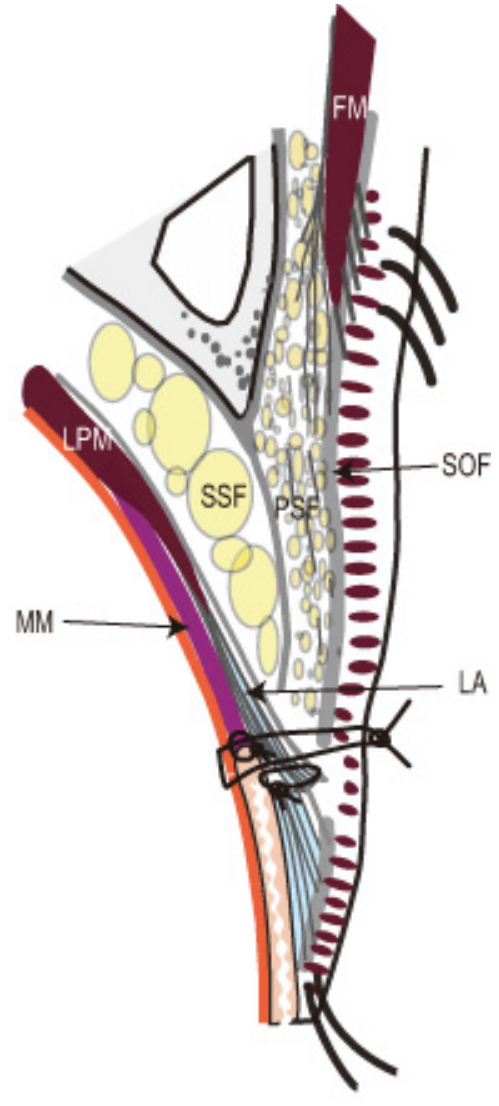

(C)

Fig. (1). The schema of the transconjunctival levator surgery. (A) Once Müller's muscle is incised, the levator aponeurosis can be reached. (B) The aponeurosis is appropriately advanced and fixed to the tarsus. (C) Müller's muscle is re-attached to the original site on the superior border of the tarsus without resection or tacking.

quite visible for several weeks or months after surgery, especially in Asians. The transconjunctival surgical approach can therefore appeal to such reluctant patients, clear their anxiety, and increase the number of patients willing to undergo this operation.

The Müller's muscle-conjunctival resection, described by Putterman and Urist [13], is a conventional transconjunctival approach in ptosis surgery. This operation is indeed simple and yields a successful correction of the eyelid height in patients with aponeurotic ptosis; however, it does not address the primary cause of aponeurotic ptosis which is a dehiscence in the attachment of the levator aponeurosis to the tarsal plate. Therefore, resecting a normal Müller's muscle $[8,13]$ and ignoring the levator aponeurosis would seem as an illogical solution for correcting aponeurotic ptosis [14]. It is also a disadvantage that the operation is generally directed toward only mild and minimal ptosis [13].

The transcutaneous advancement of the levator aponeurosis without resection of Müller's muscle is a very commonly used technique that restores the normal anatomy to the eyelid, results in good function and a naturally-looking eyelid, and with minimal eyelid lag. The transconjunctival approach for aponeurosis repair, presented here, achieves a similar outcome, and this is facilitated by leaving the lateral part of Müller's muscle intact to allow early recovery of its function. It is believed that the nerves distributed from the lateral portion along with the arcade vessels retain some function for the control of Müller's muscle [5, 15]. From our experience, there was no postoperative dysfunction of Müller's muscle; however, the period required for the eyelid to reach its final position was comparatively longer than after the transcutaneous aponeurosis repair without the resection of Müller's muscle.

A well recognized drawback of the transconjunctival approach is the fact that redundant skin in the upper eyelid could not be removed during the same operation. From our experience, however, this is not necessarily such a significant drawback. In our practice we prefer not to remove the skin of the lower part of the upper eyelid around the superior palpebral crease. The skin in this area is thin and pliable (an important esthetic feature of the upper eyelid with a beautiful fold), and when removed during blepharoplasty may sometimes result in a thicker upper eyelid with an unnatural and an artificially-looking eyelid fold. This often occurs in patients with very thick eyelids, especially in Asians. Selective removal of soft tissues, including preseptal and retro-orbicularis oculi fat (ROOF) [12], is an effective and challenging technique for attenuating the thickness of the eyelid. Subsequently, we have adopted the sub-brow skin excision technique for eliminating the redundancy of the 
(A)

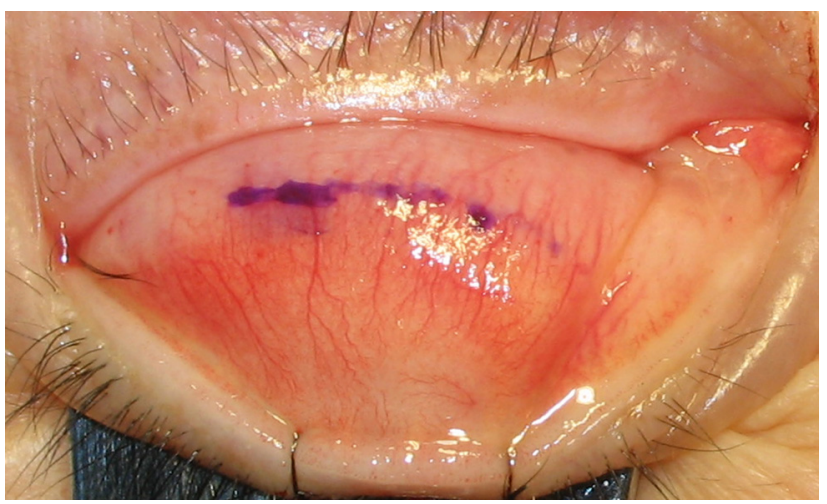

(C)

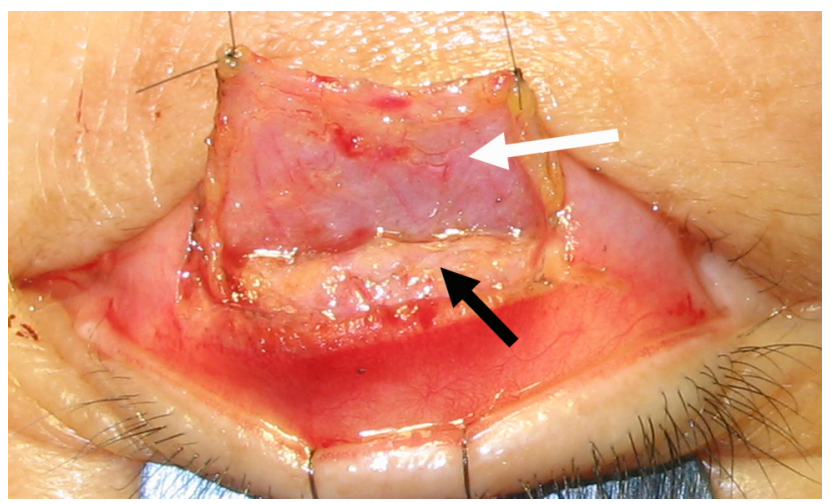

(B)

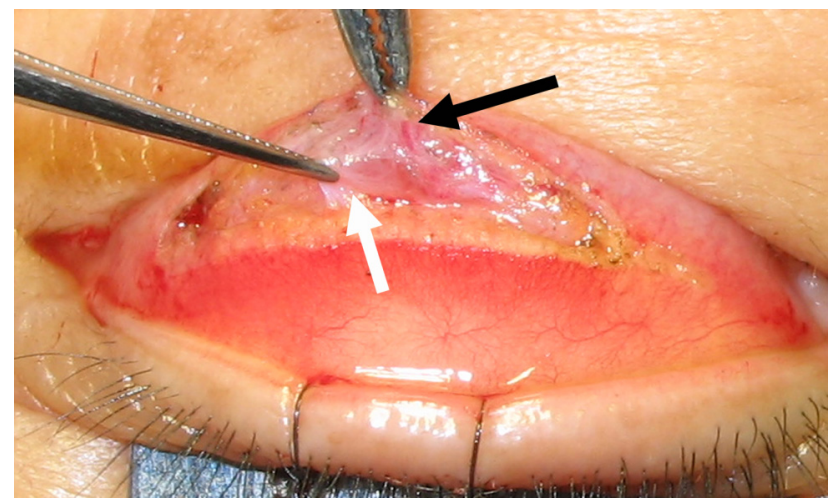

(D)

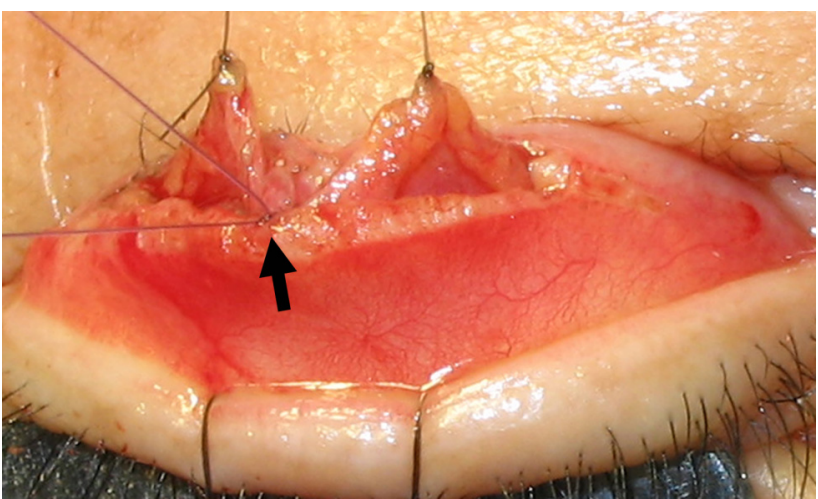

(E)

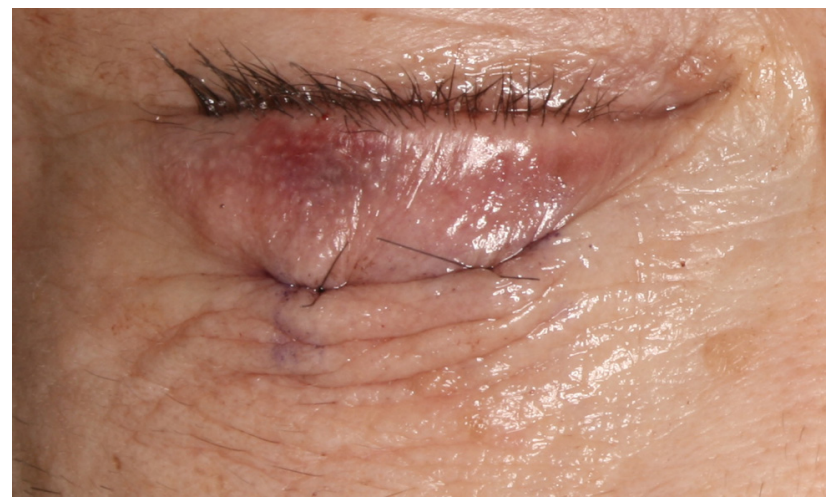

Fig. (2). (A) Conjunctival incision. (B) A very thin glossy fascia (white arrow) between the levator aponeurosis and Müller's muscle (black arrow) is helpful for identifying them. (C) Levator aponeurosis (white arrow) is incised and separated upward from the suborbicularis fascia (black arrow) and scant preseptal fat. (D) Fixation of the aponeurosis to the tarsus. (E) Nylon sutures (7-0) are placed to fix the conjunctiva to the tarsal border as well as to make a superior palpebral crease.

upper eyelid. This is a simple technique which is based on removal of the drooping thick skin in the upper eyelid while avoiding disfiguring of the original architecture of the upper eyelid crease, and obtaining a naturally-looking and youthful eyelid. The post-operative recovery time is short and the scar is easily concealed with make-up. Eventually, the scar is inconspicuous and the telltale traits of surgery are hardly recognizable in most patients. We believe that this method could become a prevalent option for reducing the redundancy of the upper eyelid in Asians.

\section{CONCLUSION}

In conclusion, the transconjunctival levator aponeurosis advancement without resection of Müller's muscle is an effective technique for blepharoptosis repair, and is associated with a low rate of complications if patients are appropriately selected. This method is quick, does not require a skin incision, results in an excellent postoperative eyelid contour, and should therefore be added to the arsenal of techniques for blepharoptosis repair.

\section{CONFLICT OF INTEREST}

The authors have no financial interest related to the manuscript. 
(A)

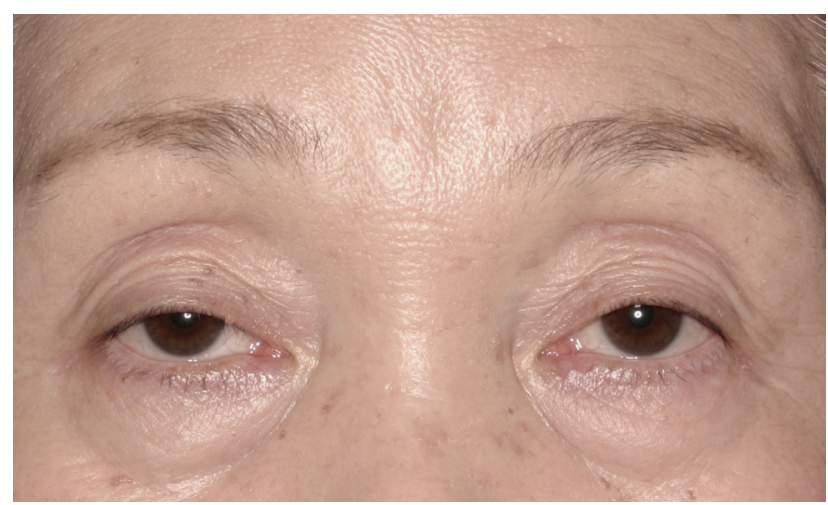

(C)

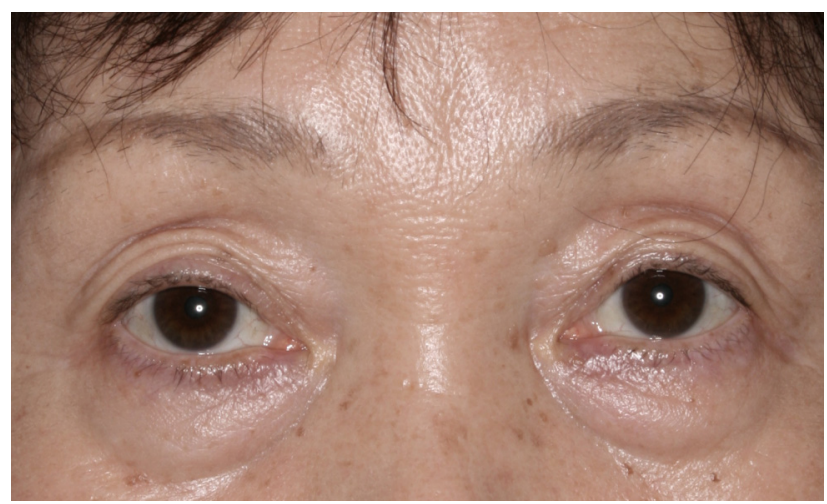

(B)

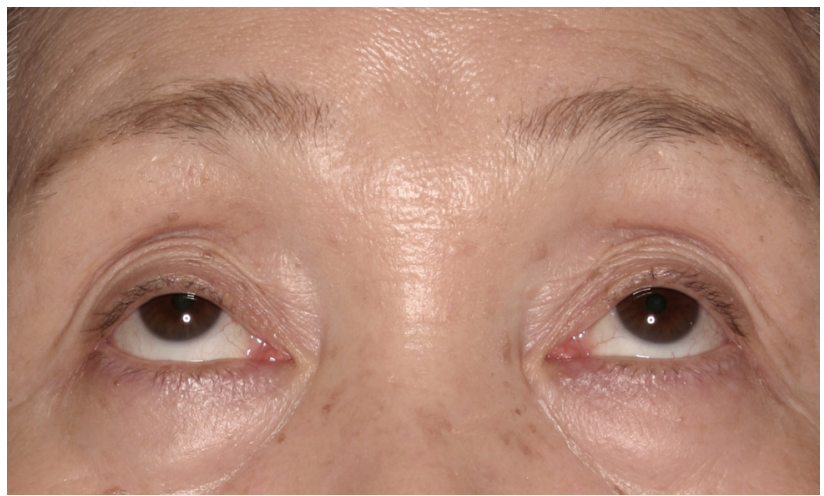

(D)

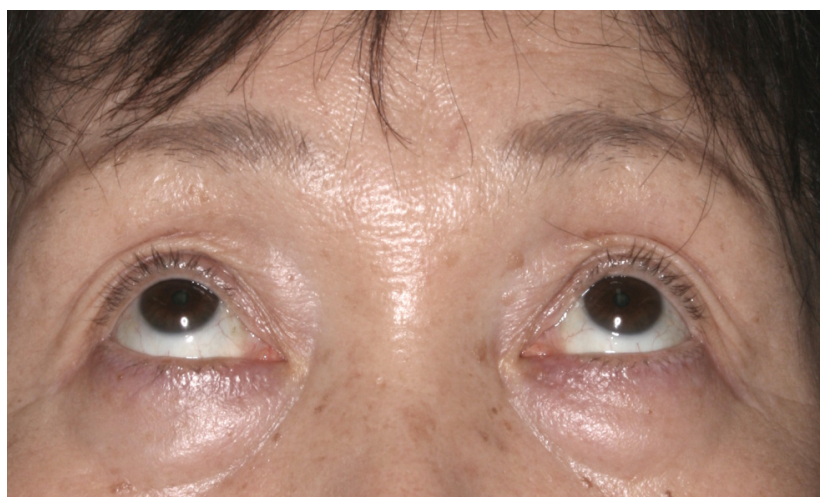

(E)

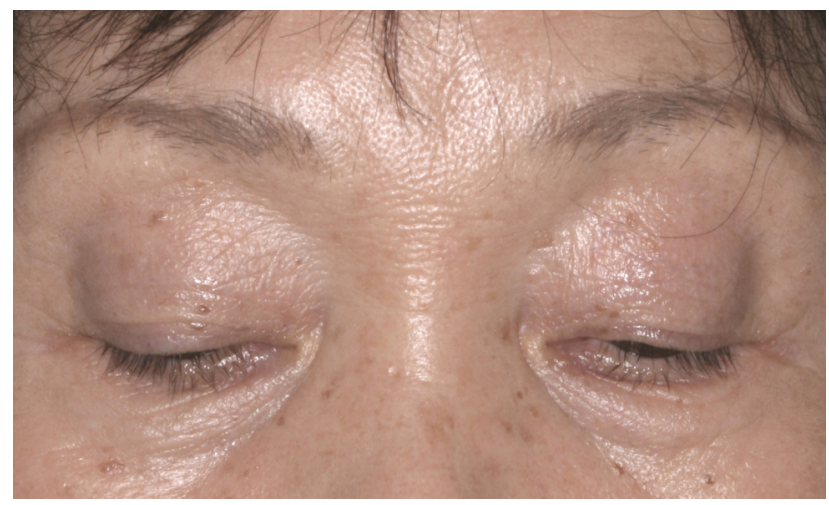

Fig. (3). (A) A 65-year-old woman with acquired bilateral blepharoptosis, straight gaze (A) and upgaze (B). Six months after correction by transconjunctival aponeurotic surgery, straight gaze $(\mathbf{C})$, upgaze $(\mathbf{D})$ and downgaze $(\mathbf{E})$.

\section{REFERENCES}

[1] Ichinose A, Tahara S. Transconjunctival levator aponeurotic repair without resection of Muller's muscle. Aesthetic Plast Surg 2007; 31: 279-84.

[2] Johnson CC. Blepharoptosis: a general consideration of surgical methods; with the results in 162 operations. Am J Ophthalmol 1954; 38: 129-62.

[3] Jones LT, Quickert MH, Wobig JL. The cure of ptosis by aponeurotic repair. Arch Ophthalmol 1975; 93: 629-34.

[4] Anderson RL, Dixon RS. Aponeurotic ptosis surgery. Arch Ophthalmol 1979; 97: 1123-8.

[5] Matsuo K. Restoration of involuntary tonic contraction of the levator muscle in patients with aponeurotic blepharoptosis or Horner syndrome by aponeurotic advancement using the orbital septum. Scand J Plast Reconstr Surg Hand Surg 2003; 37: 81-9.
[6] de Blascovics L. A new operation for ptosis with shortening of levator and tarsus. Arch Ophthalmol 1923; 52: 563-73.

[7] Berke RN. A simplified Blaskovics operation for blepharoptosis; results in ninety-one operations. AMA Arch Ophthalmol 1952; 48: 460-95.

[8] Fasanella RM, Servat J. Levator resection for minimal ptosis, with indications and reappraisal. Int Ophthalmol Clin 1970; 10: 117-30.

[9] Betharia SM. Transconjunctival levator resection: a modified simple technique. Ann Ophthalmol 1988; 20: 234-8.

[10] Mutou Y, Mutou H. Intradermal double eyelid operation and its follow-up results. Br J Plast Surg 1972; 25: 285-91.

[11] Liao WC, Tung TC, Tsai TR, Wang CY, Lin CH. Celebrity arcade suture blepharoplasty for double eyelid. Aesthetic Plast Surg 2005; 29: $540-5$.

[12] Ichinose A, Tahara S. Extended preseptal fat resection in Asian blepharoplasty. Ann Plast Surg 2008; 60: 121-6. 
[13] Putterman AM, Urist MJ. Muller muscle-conjunctiva resection. Technique for treatment of blepharoptosis. Arch Ophthalmol 1975; 93: 619-23.
[14] Callahan MA, Beard CB, Callahan M, Beard C. Beard's ptosis, $4^{\text {th }}$ ed. Birmingam: Aesculapius Publishing Company 1990.

[15] Sisler HA. Surgical correction of blepharoptosis and lower-eyelid retraction in dry-eyed patients. Arch Ophthalmol 1982; 100: 800-1.

Received: April 15, 2010

Revised: June 25, 2010

Accepted: July 19, 2010

(C) Ichinose and Leibovitch; Licensee Bentham Open.

This is an open access article licensed under the terms of the Creative Commons Attribution Non-Commercial License (http: //creativecommons.org/licenses/by$\mathrm{nc} / 3.0 /$ ) which permits unrestricted, non-commercial use, distribution and reproduction in any medium, provided the work is properly cited. 